\title{
ILUMINACIÓN EN CAMINOS, CALLES Y RUTAS. CLASIFICACIÓN Y NIVELES
}

Mg. Esp. Ing. Rubén Edgar CORVALÁN¹; Esp Ing Norberto Argentino SANABRIA; Esp. Agrim. Elvira FERRARI; Víctor Roberto SABAJ²; Hernán Ricardo FLEITAS ${ }^{2}$

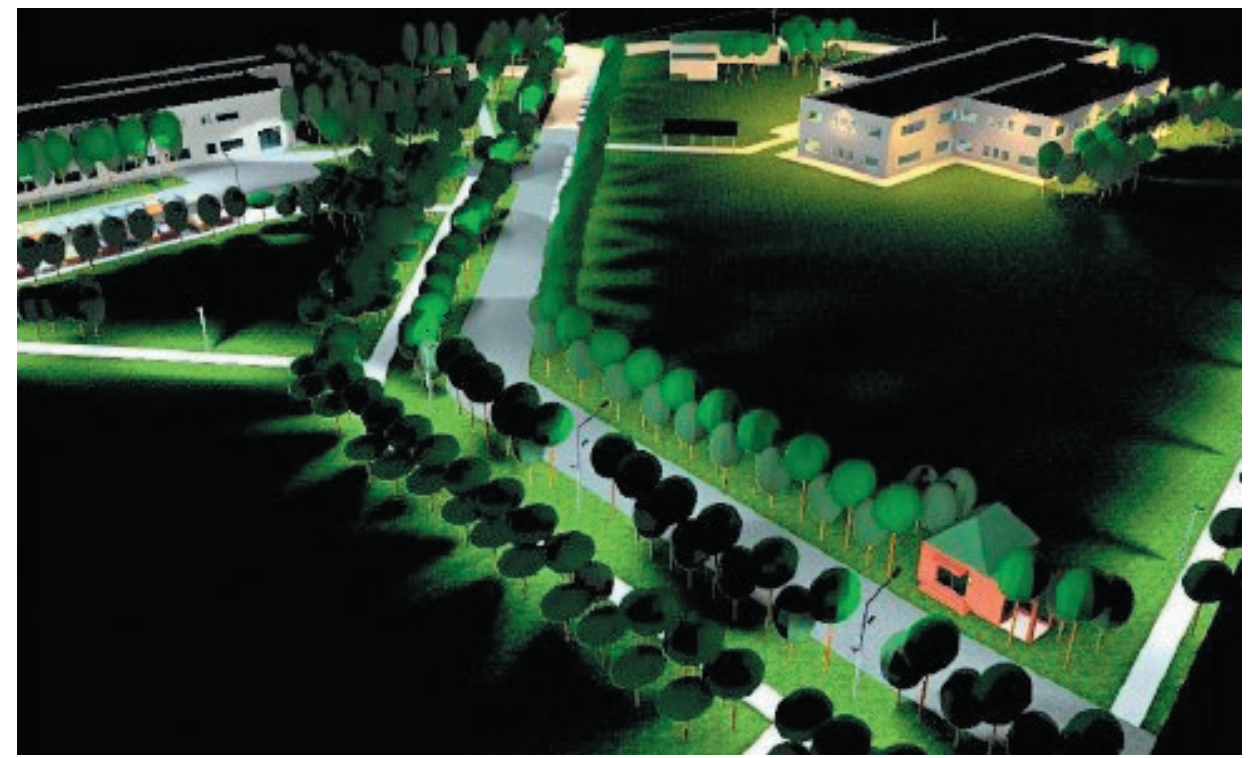

Resumen: El estudio de caso, se llevó adelante mediante el análisis de los niveles de iluminancia del acceso principal al campus Deodoro Roca, de la Universidad Nacional del Nordeste a fin de inferir a partir de estos los niveles de iluminación a considerar en calles y avenidas del nordeste argentino.

Palabras Clave: luminotecnia, luminancia, iluminancia, calles, avenidas.
Introducción: Considerando las características especiales de cada una de las vías de comunicación, pero con base a la matriz utilizada en este estudio. Primeramente, en trabajos de campo se levantaron utilizando equipamiento adecuado los datos del estado actual de la iluminación del acceso principal, y en base a ellos, se consideró si las condiciones son las establecidas por normas (IRAM AADL J2022-2010). 
Inicialmente se evaluó la calzada por el estudio, utilizando la siguiente tabla. tipo de tránsito que se tiene en el lugar de

\section{CLASIFICACION DE CALZADA}

\begin{tabular}{|c|c|c|c|}
\hline Clase & $\begin{array}{l}\text { Carácter del } \\
\text { tránsito }\end{array}$ & Descripción & Ejemplos \\
\hline $\mathrm{A}^{*}$ & $\begin{array}{l}\text { MUY RÁPIDO } \\
\mathrm{V}>100 \mathrm{~km} / \mathrm{h}\end{array}$ & $\begin{array}{l}\text { Calzadas de manos separadas, dos o más } \\
\text { carriles por mano, libre de cruces a nivel, } \\
\text { control de accesos y salidas }\end{array}$ & AUTOPISTAS \\
\hline $\mathrm{B}^{*}$ & $\begin{array}{c}\text { RÁPIDO } \\
\mathrm{V}<100 \mathrm{~km} / \mathrm{h}\end{array}$ & $\begin{array}{l}\text { Calzadas para tránsito rápido, } \\
\text { importante, sin separadores de tránsito. }\end{array}$ & $\begin{array}{l}\text { TRAMOS DE RUTAS } \\
\text { NACIONALES, } \\
\text { PROVINCIALES }\end{array}$ \\
\hline $\mathrm{C}^{* *}$ & $\begin{array}{l}\text { SEMIRÁPIDO } \\
\mathrm{V}<60 \mathrm{~km} / \mathrm{h}\end{array}$ & $\begin{array}{l}\text { Calzadas de una o dos direcciones de } \\
\text { desplazamiento, con carriles de } \\
\text { estacionamiento o sin ellos; con intensa } \\
\text { presencia de peatones y obstáculos. }\end{array}$ & $\begin{array}{l}\text { AVENIDAS PRINCIPALES } \\
\text { VÍAS DE ENLACE ENTRE } \\
\text { SECTORES IMPORTANTES }\end{array}$ \\
\hline $\mathrm{D}^{* *}$ & $\begin{array}{c}\text { LENTO } \\
\mathrm{V}<40 \mathrm{~km} / \mathrm{h}\end{array}$ & $\begin{array}{c}\text { Calzadas con desplazamiento lento y trabado; } \\
\text { con carriles de estacionamiento o sin ellos; } \\
\text { con intensa presencia de peatones y } \\
\text { obstáculos. }\end{array}$ & $\begin{array}{l}\text { ARTERIAS COMERCIALES, } \\
\text { CENTROS DE COMPRA }\end{array}$ \\
\hline$E^{* *}$ & $\begin{array}{l}\text { MODERADO } \\
\mathrm{V}<50 \mathrm{~km} / \mathrm{h}\end{array}$ & $\begin{array}{l}\text { Acumulan y conducen el tránsito desde un } \\
\text { barrio hacia vías de tránsito de orden superior, } \\
\text { (clases A, B, C, D). }\end{array}$ & $\begin{array}{l}\text { AVENIDAS SECUNDARIAS } \\
\text { CALLES COLECTORAS DE } \\
\text { TRÁNSITO }\end{array}$ \\
\hline$F^{* *}$ & $\begin{array}{c}\text { LENTO } \\
\mathrm{V}<\mathbf{4 0} \mathbf{~ k m / h}\end{array}$ & $\begin{array}{l}\text { Calles residenciales de una o dos manos; } \\
\text { con tránsito exclusivamente local. } \\
\text { Presencia de peatones y obstáculos. }\end{array}$ & CALLES RESIDENCIALES \\
\hline${ }^{*} \operatorname{Sin} 1$ & de peatones. & & \\
\hline
\end{tabular}

Según con la tabla anterior la calzada en estudio está dentro de la clase F (calles residenciales)

También debemos evaluar el estado actual de las luminarias instaladas que se pueden ver afectadas por diversas causas como las siguientes:

- Contaminación ambiental.

- Saltos térmicos bruscos y continuos.

- Variación de las tensiones de alimentación.
- Ópticas inadecuadamente diseñadas.

- Degradación natural de la lámpara.

- Tensión del arco de la lámpara.

- Propiedad reflectiva del material de la calzada.

\section{Materiales y Métodos:}

Se utilizaron dos métodos de evaluación en primera instancia el método de iluminancia (establecido por norma para este tipo de calzada) con el cual se precedió a dividir la trayectoria del acceso en 
6 (seis) tramos, y en cada uno de ellos se marcó una grilla de puntos sobre el terreno a evaluar (calzada), y posteriormente se llevó a cabo la medición de iluminancia (lux) en cada punto a una altura de 0.05 metros (como está estipulado en la norma), esta medición fue efectuada con la ayuda de un luxómetro de la marca EXTECH instruments international, acompañado por un telémetro laser y establecidos los punto con la estación total.

Para hallar la Iluminancia Horizontal (Eh): que es la iluminancia en un lugar, obtenida sobre un plano horizontal en los puntos de evaluación. El luxómetro se apoyó sobre la calzada debiendo contemplar algún mecanismo que asegure la horizontalidad del plano de medición.

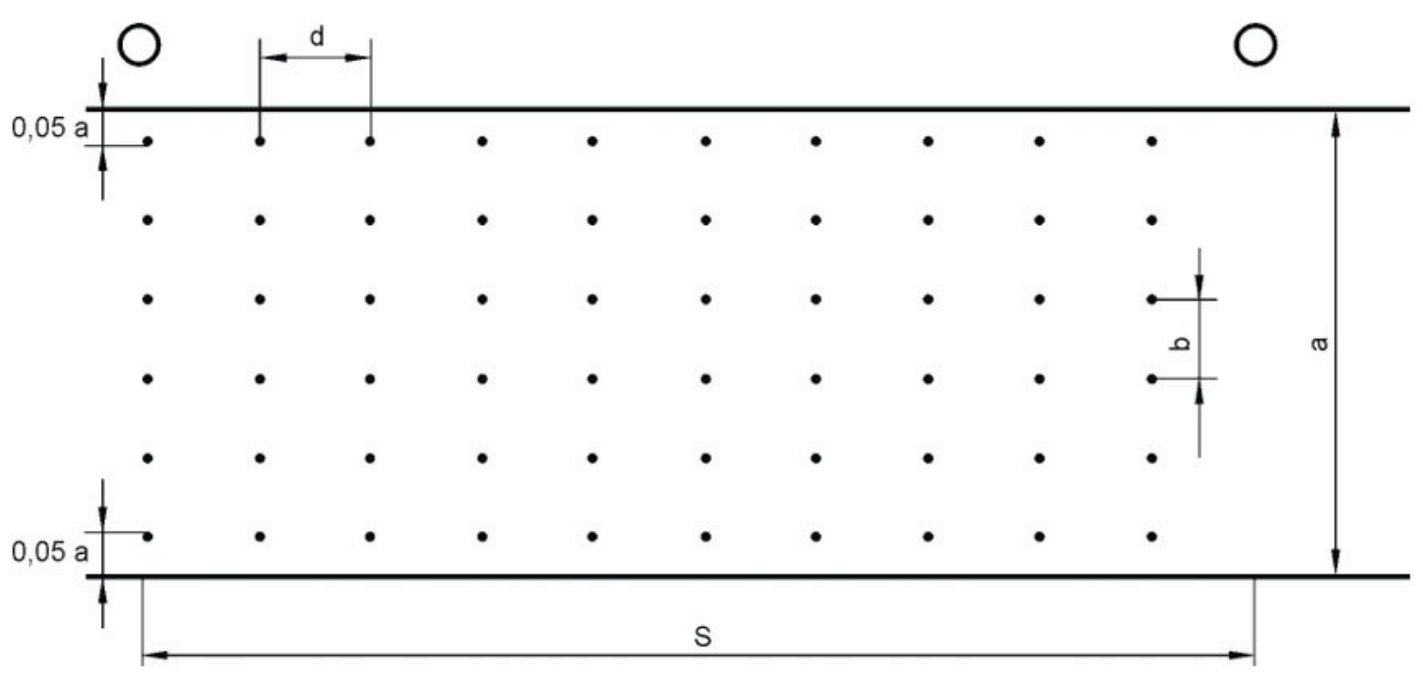

Siendo:

- a el ancho de calzada, en metros;

- d la distancia longitudinal (en metros), entre los puntos de evaluación $\mathrm{d}=$ $\mathrm{S} / \mathrm{N}$

- $\quad \mathrm{N}$ el número entero, que puede tomar los valores siguientes:

- $\mathbf{N}=10$ para $\mathrm{S} \leq 50 \mathrm{~m}$

- $\quad \mathbf{N}=$ menor número entero que permite una $\mathrm{d} \leq 5 \mathrm{~m}$ para $\mathrm{S}>50 \mathrm{mb}=0,9 \cdot \mathrm{a} / \mathrm{M}$

- b la distancia transversal (en metros), entre los puntos de evaluación;

- M el menor número entero que permite un $b \leq 1,5 \mathrm{~m}$.
Con los datos obtenidos se tabuló una tabla, con la cual se hallaron los valores de Emin (iluminancia mínima), Emax (iluminancia máxima), y Em (iluminancia media). Este trabajo fue realizado para cada tramo y provistos de estos datos se hicieron los cálculos para determinar el estado de la iluminación actual.

El segundo método que se utilizo fue con el empleo de software llamado DIALux , de fácil acceso y muy poderoso para los estudios luminotécnicos. Para proceder con este método primero se efectuó un relevamiento del ambiente considerando el 
tipo de material de la calzada y también de los alrededores como ser la vegetación, las edificaciones y los distintos tipos de suelos. También se consideró el tipo de luminaria así como las condiciones en que se encuentran las mismas, y con estos datos se procedió a renderizar (modelado en $3 \mathrm{D}$ ), el terreno en el software. Los pasos que se llevaron a cabo para tener una máxima aproximación fueron:

- Ubicar la zona en una imagen satelital.

- Hacer una captura de pantalla de la misma para poder utilizar las medidas exactas.

- Esta imagen se la inserto en el software autoCAD para poder hacer el trazado de la calzada sobre ella.

- En el campo se hizo la medición de las distancias entre luminarias y entre la luminaria y el terreno en estudio.

- Los datos validos del plano obtenido en autoCAD, se procedieron a colocarlo en el software DIALux.

- Dentro del programa se volvió a trazar la escena del plano de autoCAD y colocaron los distintos tipos de materiales y todos los objetos más relevantes.

- Se procedió a instalar las luminarias de los catálogos provistos por el software, también se introdujeron especificaciones como altura de montaje y ángulo de rotación de la misma.

- Se coloco una grilla de puntos de medición a la misma altura y con la misma disposición que en el campo.

- Se procedió a hacer la simulación con todos los parámetros ya colocados, y cuyos datos fueron obtenidos.

NOTA: dicho software toma en cuenta la curva isolux de la luminaria propiamente dicha con la lámpara seleccionada para esa luminaria y obtiene a si una curva total. En el gráfico siguiente se muestra un ejemplo de curva isolux hallada.

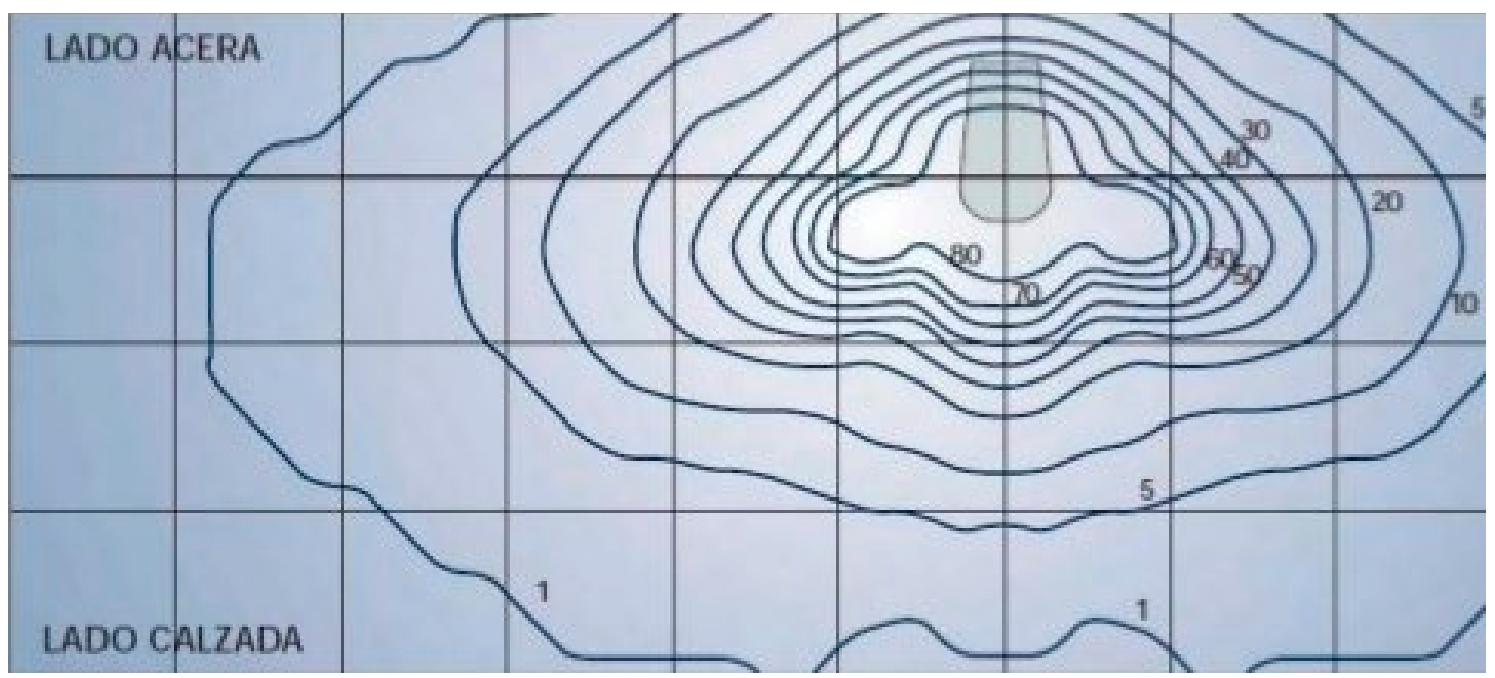

DATOS OBTENIDOS DEL REELEVAMIENTO EN CAMPO 


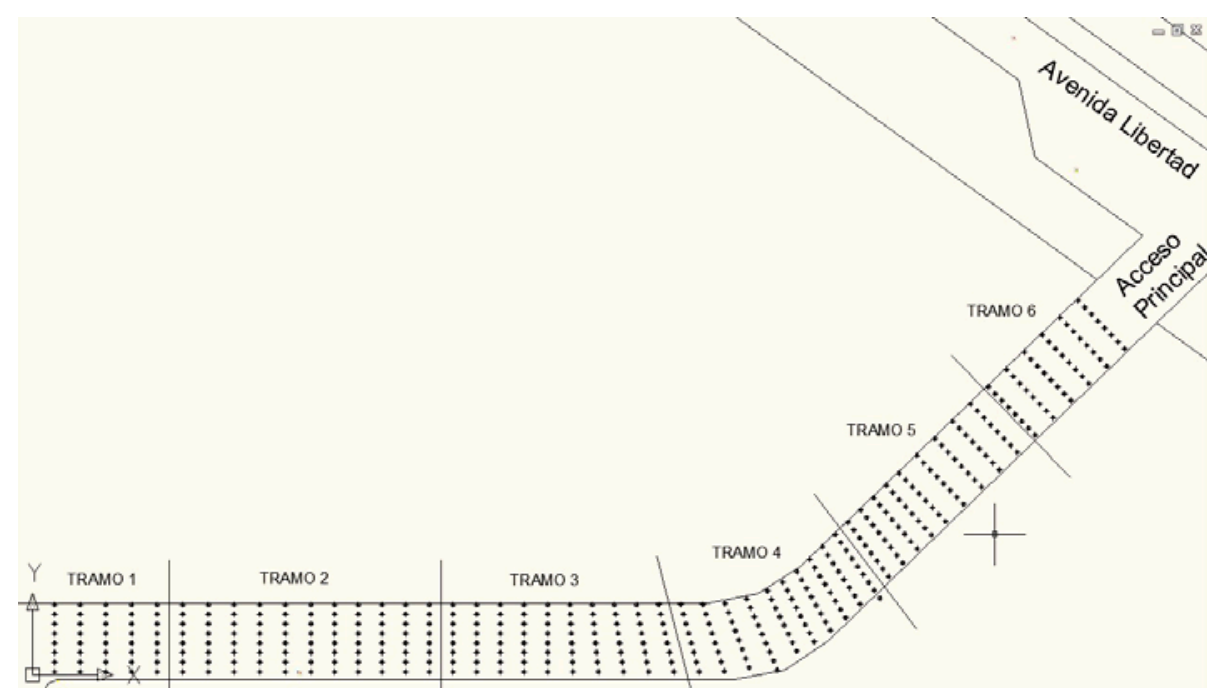

\begin{tabular}{|c|c|c|c|c|c|c|c|c|c|c|}
\hline \multicolumn{11}{|c|}{$\begin{array}{c}\text { TRAMO } 1 \\
\mathrm{~h}=9.09 \mathrm{~m}-\text { Separación entre luminarias }=36 \mathrm{~m}\end{array}$} \\
\hline 0 & 0 & $\mathbf{0}$ & 0 & 1,076 & 1,076 & 0 & 1,076 & 2,152 & 2,152 & 2,152 \\
\hline 0 & 0 & 0 & 0 & 0 & 2,152 & 0 & 1,076 & 3,228 & 6,456 & 8,608 \\
\hline 0 & 1,076 & $\mathbf{0}$ & 0 & 0 & 0 & 0 & 1,076 & 4,304 & 11,84 & 17,22 \\
\hline 0 & 2,152 & $\mathbf{0}$ & $\mathbf{0}$ & 0 & 0 & 0 & 2,152 & 4,304 & 15,07 & 24,75 \\
\hline 0 & 2,152 & $\mathbf{0}$ & 0 & 0 & 0 & 1,076 & 2,152 & 6,456 & 19,37 & 35,51 \\
\hline 1,076 & 3,228 & $\mathbf{0}$ & 0 & 0 & $\mathbf{0}$ & 0 & 2,152 & 6,456 & 26,9 & 47,34 \\
\hline 1,076 & 5,38 & $\mathbf{0}$ & 0 & 0 & 0 & 0 & 2,152 & 8,608 & 33,36 & 59,18 \\
\hline 3,228 & 0 & 0 & 0 & 0 & 0 & 0 & 2,152 & 11,84 & 39,81 & 60,26 \\
\hline 4,304 & 0 & 0 & O & 0 & 0 & 0 & 2,152 & 11,84 & 48,42 & 60,26 \\
\hline
\end{tabular}

\begin{tabular}{|c|c|c|}
\hline Emin & Emax & Em \\
\hline 0 & 60,256 & 6,29302 \\
\hline G1 = Emin/Em & \multicolumn{2}{|c|}{0} \\
\hline G2=Emin/Emax & \multicolumn{2}{|c|}{0} \\
\hline
\end{tabular}

\begin{tabular}{|c|c|c|c|c|c|c|c|c|c|c|}
\hline \multicolumn{10}{|c|}{ TRAMO 2 } \\
\hline \multicolumn{10}{|c|}{ h=9.09m - Separación entre luminarias=29.4m } \\
\hline $\mathbf{1 , 0 7 6}$ & $\mathbf{1 , 0 7 6}$ & $\mathbf{1 , 0 7 6}$ & $\mathbf{0}$ & $\mathbf{0}$ & $\mathbf{0}$ & $\mathbf{0}$ & $\mathbf{1 , 0 7 6}$ & $\mathbf{1 , 0 7 6}$ & $\mathbf{0}$ & $\mathbf{2 , 1 5 2}$ \\
\hline $\mathbf{1 , 0 7 6}$ & $\mathbf{2 , 1 5 2}$ & $\mathbf{1 , 0 7 6}$ & $\mathbf{0}$ & $\mathbf{0}$ & $\mathbf{0}$ & $\mathbf{0}$ & $\mathbf{1 , 0 7 6}$ & $\mathbf{1 , 0 7 6}$ & $\mathbf{2 , 1 5 2}$ & $\mathbf{2 , 1 5 2}$ \\
\hline $\mathbf{1 , 0 7 6}$ & $\mathbf{2 , 1 5 2}$ & $\mathbf{2 , 1 5 2}$ & $\mathbf{0}$ & $\mathbf{0}$ & $\mathbf{0}$ & $\mathbf{0}$ & $\mathbf{1 , 0 7 6}$ & $\mathbf{1 , 0 7 6}$ & $\mathbf{2 , 1 5 2}$ & $\mathbf{3 , 2 2 8}$ \\
\hline $\mathbf{1 , 0 7 6}$ & $\mathbf{4 , 3 0 4}$ & $\mathbf{2 , 1 5 2}$ & $\mathbf{0}$ & $\mathbf{0}$ & $\mathbf{0}$ & $\mathbf{0}$ & $\mathbf{1 , 0 7 6}$ & $\mathbf{2 , 1 5 2}$ & $\mathbf{3 , 2 2 8}$ & $\mathbf{1 , 0 7 6}$ \\
\hline $\mathbf{1 , 0 7 6}$ & $\mathbf{6 , 4 5 6}$ & $\mathbf{0}$ & $\mathbf{1 , 0 7 6}$ & $\mathbf{0}$ & $\mathbf{0}$ & $\mathbf{0}$ & $\mathbf{1 , 0 7 6}$ & $\mathbf{2 , 1 5 2}$ & $\mathbf{4 , 3 0 4}$ & $\mathbf{2 , 1 5 2}$ \\
\hline $\mathbf{3 , 2 2 8}$ & $\mathbf{0}$ & $\mathbf{0}$ & $\mathbf{0}$ & $\mathbf{0}$ & $\mathbf{0}$ & $\mathbf{0}$ & $\mathbf{1 , 0 7 6}$ & $\mathbf{2 , 1 5 2}$ & $\mathbf{4 , 3 0 4}$ & $\mathbf{7 , 5 3 2}$ \\
\hline $\mathbf{1 , 0 7 6}$ & $\mathbf{1 1 , 8 4}$ & $\mathbf{5 , 3 8}$ & $\mathbf{1 , 0 7 6}$ & $\mathbf{0}$ & $\mathbf{0}$ & $\mathbf{0}$ & $\mathbf{0}$ & $\mathbf{2 , 1 5 2}$ & $\mathbf{5 , 3 8}$ & $\mathbf{1 1 , 8 4}$ \\
\hline $\mathbf{0}$ & $\mathbf{1 5 , 0 6}$ & $\mathbf{6 , 4 5 6}$ & $\mathbf{0}$ & $\mathbf{0}$ & $\mathbf{0}$ & $\mathbf{0}$ & $\mathbf{0}$ & $\mathbf{2 , 1 5 2}$ & $\mathbf{7 , 5 3 2}$ & $\mathbf{7 , 5 3 2}$ \\
\hline $\mathbf{2 3 , 6 7 2}$ & $\mathbf{1 5 , 0 6}$ & $\mathbf{0}$ & $\mathbf{0}$ & $\mathbf{0}$ & $\mathbf{0}$ & $\mathbf{0}$ & $\mathbf{0}$ & $\mathbf{0}$ & $\mathbf{0}$ & $\mathbf{7 , 5 3 2}$ \\
\hline
\end{tabular}




\begin{tabular}{|c|c|c|}
\hline Emin & Emax & Em \\
\hline 0 & 23,672 & $\mathbf{2 , 0 8 6 7 8 8}$ \\
\hline G1 = Emin/Em & \multicolumn{2}{|c|}{0} \\
\hline G2=Emin/Emax & \multicolumn{2}{|c|}{0} \\
\hline
\end{tabular}

\begin{tabular}{|c|c|c|c|c|c|c|c|c|c|c|}
\hline \multicolumn{10}{|c|}{ TRAMO 3 } \\
\hline \multicolumn{10}{|c|}{ h=99m - Separación entre luminarias=26.8m } \\
\hline $\mathbf{2 , 1 5 2}$ & $\mathbf{2 , 1 5 2}$ & $\mathbf{1 , 0 7 6}$ & $\mathbf{1 , 0 7 6}$ & $\mathbf{1 , 0 7 6}$ & $\mathbf{1 , 0 7 6}$ & $\mathbf{1 , 0 7 6}$ & $\mathbf{1 , 0 7 6}$ & $\mathbf{1 , 0 7 6}$ & $\mathbf{1 , 0 7 6}$ & $\mathbf{1 , 0 7 6}$ \\
\hline $\mathbf{2 , 1 5 2}$ & $\mathbf{2 , 1 5 2}$ & $\mathbf{1 , 0 7 6}$ & $\mathbf{1 , 0 7 6}$ & $\mathbf{1 , 0 7 6}$ & $\mathbf{1 , 0 7 6}$ & $\mathbf{1 , 0 7 6}$ & $\mathbf{1 , 0 7 6}$ & $\mathbf{1 , 0 7 6}$ & $\mathbf{2 , 1 5 2}$ & $\mathbf{1 , 0 7 6}$ \\
\hline $\mathbf{2 , 1 5 2}$ & $\mathbf{2 , 1 5 2}$ & $\mathbf{1 , 0 7 6}$ & $\mathbf{1 , 0 7 6}$ & $\mathbf{1 , 0 7 6}$ & $\mathbf{1 , 0 7 6}$ & $\mathbf{1 , 0 7 6}$ & $\mathbf{1 , 0 7 6}$ & $\mathbf{2 , 1 5 2}$ & $\mathbf{2 , 1 5 2}$ & $\mathbf{2 , 1 5 2}$ \\
\hline $\mathbf{3 , 2 2 8}$ & $\mathbf{2 , 1 5 2}$ & $\mathbf{2 , 1 5 2}$ & $\mathbf{1 , 0 7 6}$ & $\mathbf{1 , 0 7 6}$ & $\mathbf{1 , 0 7 6}$ & $\mathbf{1 , 0 7 6}$ & $\mathbf{1 , 0 7 6}$ & $\mathbf{2 , 1 5 2}$ & $\mathbf{2 , 1 5 2}$ & $\mathbf{2 , 1 5 2}$ \\
\hline $\mathbf{3 , 2 2 8}$ & $\mathbf{2 , 1 5 2}$ & $\mathbf{2 , 1 5 2}$ & $\mathbf{1 , 0 7 6}$ & $\mathbf{1 , 0 7 6}$ & $\mathbf{1 , 0 7 6}$ & $\mathbf{1 , 0 7 6}$ & $\mathbf{1 , 0 7 6}$ & $\mathbf{3 , 2 2 8}$ & $\mathbf{3 , 2 2 8}$ & $\mathbf{3 , 2 2 8}$ \\
\hline $\mathbf{3 , 2 2 8}$ & $\mathbf{3 , 2 2 8}$ & $\mathbf{2 , 1 5 2}$ & $\mathbf{1 , 0 7 6}$ & $\mathbf{1 , 0 7 6}$ & $\mathbf{1 , 0 7 6}$ & $\mathbf{1 , 0 7 6}$ & $\mathbf{2 , 1 5 2}$ & $\mathbf{3 , 2 2 8}$ & $\mathbf{3 , 2 2 8}$ & $\mathbf{3 , 2 2 8}$ \\
\hline $\mathbf{3 , 2 2 8}$ & $\mathbf{3 , 2 2 8}$ & $\mathbf{3 , 2 2 8}$ & $\mathbf{1 , 0 7 6}$ & $\mathbf{1 , 0 7 6}$ & $\mathbf{1 , 0 7 6}$ & $\mathbf{1 , 0 7 6}$ & $\mathbf{2 , 1 5 2}$ & $\mathbf{4 , 3 0 4}$ & $\mathbf{4 , 3 0 4}$ & $\mathbf{4 , 3 0 4}$ \\
\hline $\mathbf{3 , 2 2 8}$ & $\mathbf{5 , 3 8}$ & $\mathbf{4 , 3 0 4}$ & $\mathbf{1 , 0 7 6}$ & $\mathbf{0}$ & $\mathbf{1 , 0 7 6}$ & $\mathbf{2 , 1 5 2}$ & $\mathbf{3 , 2 2 8}$ & $\mathbf{5 , 3 8}$ & $\mathbf{6 , 4 5 6}$ & $\mathbf{6 , 4 5 6}$ \\
\hline $\mathbf{3 , 2 2 8}$ & $\mathbf{6 , 4 5 6}$ & $\mathbf{4 , 3 0 4}$ & $\mathbf{1 , 0 7 6}$ & $\mathbf{0}$ & $\mathbf{1 , 0 7 6}$ & $\mathbf{2 , 1 5 2}$ & $\mathbf{3 , 2 2 8}$ & $\mathbf{6 , 4 5 6}$ & $\mathbf{8 , 6 0 8}$ & $\mathbf{8 , 6 0 8}$ \\
\hline
\end{tabular}

\begin{tabular}{|c|c|c|}
\hline Emin & Emax & Em \\
\hline 0 & 8,608 & $\mathbf{2 , 2 8 2 4 2 4}$ \\
\hline G1 = Emin/Em & \multicolumn{2}{|c|}{0} \\
\hline G2=Emin/Emax & \multicolumn{2}{|c|}{0} \\
\hline
\end{tabular}

\begin{tabular}{|c|c|c|c|c|c|c|c|c|c|c|}
\hline \multicolumn{10}{|c|}{ TRAMO 4 } \\
\hline $\mathbf{1 0}=9,09 m$ & Separación entre luminarias=28m \\
\hline $\mathbf{3 , 2 2 8}$ & $\mathbf{3 , 2 2 8}$ & $\mathbf{1 , 0 7 6}$ & $\mathbf{1 , 0 7 6}$ & $\mathbf{0}$ & $\mathbf{0}$ & $\mathbf{0}$ & $\mathbf{0}$ & $\mathbf{0}$ & $\mathbf{0}$ & $\mathbf{0}$ \\
\hline $\mathbf{4 , 3 0 4}$ & $\mathbf{3 , 2 2 8}$ & $\mathbf{1 , 0 7 6}$ & $\mathbf{1 , 0 7 6}$ & $\mathbf{0}$ & $\mathbf{0}$ & $\mathbf{0}$ & $\mathbf{0}$ & $\mathbf{0}$ & $\mathbf{0}$ & $\mathbf{1 , 0 7 6}$ \\
\hline $\mathbf{4 , 3 0 4}$ & $\mathbf{3 , 2 2 8}$ & $\mathbf{1 , 0 7 6}$ & $\mathbf{1 , 0 7 6}$ & $\mathbf{0}$ & $\mathbf{0}$ & $\mathbf{0}$ & $\mathbf{0}$ & $\mathbf{0}$ & $\mathbf{1 , 0 7 6}$ & $\mathbf{1 , 0 7 6}$ \\
\hline $\mathbf{4 , 3 0 4}$ & $\mathbf{4 , 3 0 4}$ & $\mathbf{2 , 1 5 2}$ & $\mathbf{1 , 0 7 6}$ & $\mathbf{0}$ & $\mathbf{0}$ & $\mathbf{0}$ & $\mathbf{0}$ & $\mathbf{0}$ & $\mathbf{1 , 0 7 6}$ & $\mathbf{1 , 0 7 6}$ \\
\hline $\mathbf{5 , 3 8}$ & $\mathbf{4 , 3 0 4}$ & $\mathbf{2 , 1 5 2}$ & $\mathbf{1 , 0 7 6}$ & $\mathbf{0}$ & $\mathbf{0}$ & $\mathbf{0}$ & $\mathbf{0}$ & $\mathbf{1 , 0 7 6}$ & $\mathbf{1 , 0 7 6}$ & $\mathbf{2 , 1 5 2}$ \\
\hline $\mathbf{6 , 4 5 6}$ & $\mathbf{5 , 3 8}$ & $\mathbf{3 , 2 2 8}$ & $\mathbf{1 , 0 7 6}$ & $\mathbf{0}$ & $\mathbf{0}$ & $\mathbf{0}$ & $\mathbf{0}$ & $\mathbf{1 , 0 7 6}$ & $\mathbf{1 , 0 7 6}$ & $\mathbf{2 , 1 5 2}$ \\
\hline $\mathbf{6 , 4 5 6}$ & $\mathbf{6 , 4 5 6}$ & $\mathbf{3 , 2 2 8}$ & $\mathbf{1 , 0 7 6}$ & $\mathbf{0}$ & $\mathbf{0}$ & $\mathbf{0}$ & $\mathbf{0}$ & $\mathbf{1 , 0 7 6}$ & $\mathbf{1 , 0 7 6}$ & $\mathbf{5 , 3 8}$ \\
\hline $\mathbf{9 , 6 8 4}$ & $\mathbf{7 , 5 3 2}$ & $\mathbf{4 , 3 0 4}$ & $\mathbf{1 , 0 7 6}$ & $\mathbf{0}$ & $\mathbf{0}$ & $\mathbf{0}$ & $\mathbf{0}$ & $\mathbf{1 , 0 7 6}$ & $\mathbf{1 , 0 7 6}$ & $\mathbf{4 , 3 0 4}$ \\
\hline 12,912 & $\mathbf{9 , 6 8 4}$ & $\mathbf{4 , 3 0 4}$ & $\mathbf{1 , 0 7 6}$ & $\mathbf{0}$ & $\mathbf{0}$ & $\mathbf{0}$ & $\mathbf{1 , 0 7 6}$ & $\mathbf{2 , 1 5 2}$ & $\mathbf{2 , 1 5 2}$ & $\mathbf{6 , 4 5 6}$ \\
\hline
\end{tabular}

\begin{tabular}{|c|c|c|}
\hline Emin & Emax & Em \\
\hline 0 & 12,192 & 1,782465 \\
\hline G1 = Emin/Em & \multicolumn{2}{|c|}{0} \\
\hline G2=Emin/Emax & \multicolumn{2}{|c|}{0} \\
\hline
\end{tabular}


TRAMO 5

$h=9.09 \mathrm{~m}$ - Separación entre luminarias $=22.1 \mathrm{~m}$

\begin{tabular}{|c|c|c|c|c|c|c|c|c|c|c|}
\hline $\mathbf{0}$ & $\mathbf{0}$ & $\mathbf{0}$ & $\mathbf{0}$ & $\mathbf{0}$ & $\mathbf{0}$ & $\mathbf{0}$ & $\mathbf{0}$ & $\mathbf{0}$ & $\mathbf{0}$ & $\mathbf{0}$ \\
\hline $\mathbf{0}$ & $\mathbf{0}$ & $\mathbf{0}$ & $\mathbf{0}$ & $\mathbf{0}$ & $\mathbf{0}$ & $\mathbf{0}$ & $\mathbf{0}$ & $\mathbf{0}$ & $\mathbf{0}$ & $\mathbf{0}$ \\
\hline $\mathbf{0}$ & $\mathbf{0}$ & $\mathbf{0}$ & $\mathbf{0}$ & $\mathbf{0}$ & $\mathbf{0}$ & $\mathbf{0}$ & $\mathbf{0}$ & $\mathbf{0}$ & $\mathbf{0}$ & $\mathbf{0}$ \\
\hline $\mathbf{0}$ & $\mathbf{0}$ & $\mathbf{0}$ & $\mathbf{0}$ & $\mathbf{0}$ & $\mathbf{0}$ & $\mathbf{0}$ & $\mathbf{0}$ & $\mathbf{1 , 0 7 6}$ & $\mathbf{1 , 0 7 6}$ & $\mathbf{0}$ \\
\hline $\mathbf{0}$ & $\mathbf{0}$ & $\mathbf{0}$ & $\mathbf{0}$ & $\mathbf{0}$ & $\mathbf{0}$ & $\mathbf{0}$ & $\mathbf{0}$ & $\mathbf{1 , 0 7 6}$ & $\mathbf{1 , 0 7 6}$ & $\mathbf{0}$ \\
\hline $\mathbf{0}$ & $\mathbf{0}$ & $\mathbf{0}$ & $\mathbf{0}$ & $\mathbf{0}$ & $\mathbf{0}$ & $\mathbf{0}$ & $\mathbf{1 , 0 7 6}$ & $\mathbf{1 , 0 7 6}$ & $\mathbf{2 , 1 5 2}$ & $\mathbf{1 , 0 7 6}$ \\
\hline $\mathbf{0}$ & $\mathbf{0}$ & $\mathbf{0}$ & $\mathbf{0}$ & $\mathbf{0}$ & $\mathbf{0}$ & $\mathbf{0}$ & $\mathbf{1 , 0 7 6}$ & $\mathbf{2 , 1 5 2}$ & $\mathbf{3 , 2 2 8}$ & $\mathbf{1 , 0 7 6}$ \\
\hline $\mathbf{0}$ & $\mathbf{0}$ & $\mathbf{0}$ & $\mathbf{0}$ & $\mathbf{0}$ & $\mathbf{0}$ & $\mathbf{0}$ & $\mathbf{2 , 1 5 2}$ & $\mathbf{3 , 2 2 8}$ & $\mathbf{4 , 3 0 4}$ & $\mathbf{2 , 1 5 2}$ \\
\hline $\mathbf{0}$ & $\mathbf{0}$ & $\mathbf{0}$ & $\mathbf{0}$ & $\mathbf{0}$ & $\mathbf{0}$ & $\mathbf{0}$ & $\mathbf{2 , 1 5 2}$ & $\mathbf{4 , 3 0 4}$ & $\mathbf{6 , 4 5 6}$ & $\mathbf{3 , 2 2 8}$ \\
\hline
\end{tabular}

\begin{tabular}{|c|c|c|}
\hline Emin & Emax & Em \\
\hline 0 & 6,456 & 0,456485 \\
\hline G1 = Emin/Em & \multicolumn{2}{|c|}{0} \\
\hline G2=Emin/Emax & \multicolumn{2}{|c|}{0} \\
\hline
\end{tabular}

\begin{tabular}{|c|c|c|c|c|c|c|c|c|c|c|}
\hline \multicolumn{10}{|c|}{ TRAMO 6 } \\
\hline \multicolumn{10}{|c|}{ h=9.09m - Separación entre luminarias=28m } \\
\hline $\mathbf{0}$ & $\mathbf{0}$ & $\mathbf{0}$ & $\mathbf{0}$ & $\mathbf{0}$ & $\mathbf{0}$ & $\mathbf{0}$ & $\mathbf{0}$ & $\mathbf{0}$ & $\mathbf{1 , 0 7 6}$ & $\mathbf{1 , 0 7 6}$ \\
\hline $\mathbf{0}$ & $\mathbf{0}$ & $\mathbf{0}$ & $\mathbf{0}$ & $\mathbf{0}$ & $\mathbf{0}$ & $\mathbf{0}$ & $\mathbf{0}$ & $\mathbf{1 , 0 7 6}$ & $\mathbf{2 , 1 5 2}$ & $\mathbf{2 , 1 5 2}$ \\
\hline $\mathbf{0}$ & $\mathbf{0}$ & $\mathbf{0}$ & $\mathbf{0}$ & $\mathbf{0}$ & $\mathbf{0}$ & $\mathbf{0}$ & $\mathbf{0}$ & $\mathbf{1 , 0 7 6}$ & $\mathbf{2 , 1 5 2}$ & $\mathbf{2 , 1 5 2}$ \\
\hline $\mathbf{0}$ & $\mathbf{0}$ & $\mathbf{1 , 0 7 6}$ & $\mathbf{0}$ & $\mathbf{0}$ & $\mathbf{0}$ & $\mathbf{0}$ & $\mathbf{1 , 0 7 6}$ & $\mathbf{2 , 1 5 2}$ & $\mathbf{3 , 2 2 8}$ & $\mathbf{2 , 1 5 2}$ \\
\hline $\mathbf{0}$ & 1,076 & $\mathbf{1 , 0 7 6}$ & $\mathbf{0}$ & $\mathbf{0}$ & $\mathbf{0}$ & $\mathbf{0}$ & $\mathbf{1 , 0 7 6}$ & $\mathbf{3 , 2 2 8}$ & $\mathbf{4 , 3 0 4}$ & $\mathbf{3 , 2 2 8}$ \\
\hline 1,076 & $\mathbf{2 , 1 5 2}$ & $\mathbf{1 , 0 7 6}$ & $\mathbf{0}$ & $\mathbf{0}$ & $\mathbf{0}$ & $\mathbf{0}$ & $\mathbf{1 , 0 7 6}$ & $\mathbf{3 , 2 2 8}$ & $\mathbf{5 , 3 8}$ & $\mathbf{4 , 3 0 4}$ \\
\hline 1,076 & $\mathbf{3 , 2 2 8}$ & $\mathbf{1 , 0 7 6}$ & $\mathbf{0}$ & $\mathbf{0}$ & $\mathbf{0}$ & $\mathbf{0}$ & $\mathbf{1 , 0 7 6}$ & $\mathbf{4 , 3 0 4}$ & $\mathbf{6 , 4 5 6}$ & $\mathbf{4 , 3 0 4}$ \\
\hline $\mathbf{2 , 1 5 2}$ & $\mathbf{3 , 2 2 8}$ & $\mathbf{1 , 0 7 6}$ & $\mathbf{1 , 0 7 6}$ & $\mathbf{0}$ & $\mathbf{0}$ & $\mathbf{0}$ & $\mathbf{2 , 1 5 2}$ & $\mathbf{4 , 3 0 4}$ & $\mathbf{8 , 6 0 8}$ & $\mathbf{5 , 3 8}$ \\
\hline $\mathbf{3 , 2 2 8}$ & $\mathbf{4 , 3 0 4}$ & $\mathbf{1 , 0 7 6}$ & $\mathbf{1 , 0 7 6}$ & $\mathbf{0}$ & $\mathbf{0}$ & $\mathbf{0}$ & $\mathbf{2 , 1 5 2}$ & $\mathbf{1 , 0 7 6}$ & $\mathbf{8 , 6 0 8}$ & $\mathbf{7 , 5 3 6}$ \\
\hline
\end{tabular}

\begin{tabular}{|c|c|c|}
\hline Emin & Emax & Em \\
\hline 0 & $\mathbf{8 , 6 0 8}$ & $\mathbf{1 , 3 4 7 7 5 8}$ \\
\hline G1 = Emin/Em & \multicolumn{2}{|c|}{0} \\
\hline G2=Emin/Emax & \multicolumn{2}{|c|}{0} \\
\hline
\end{tabular}




\section{REPRESENTACION EN 3 D DE LOS DATOS ANTES OBTENIDOS}

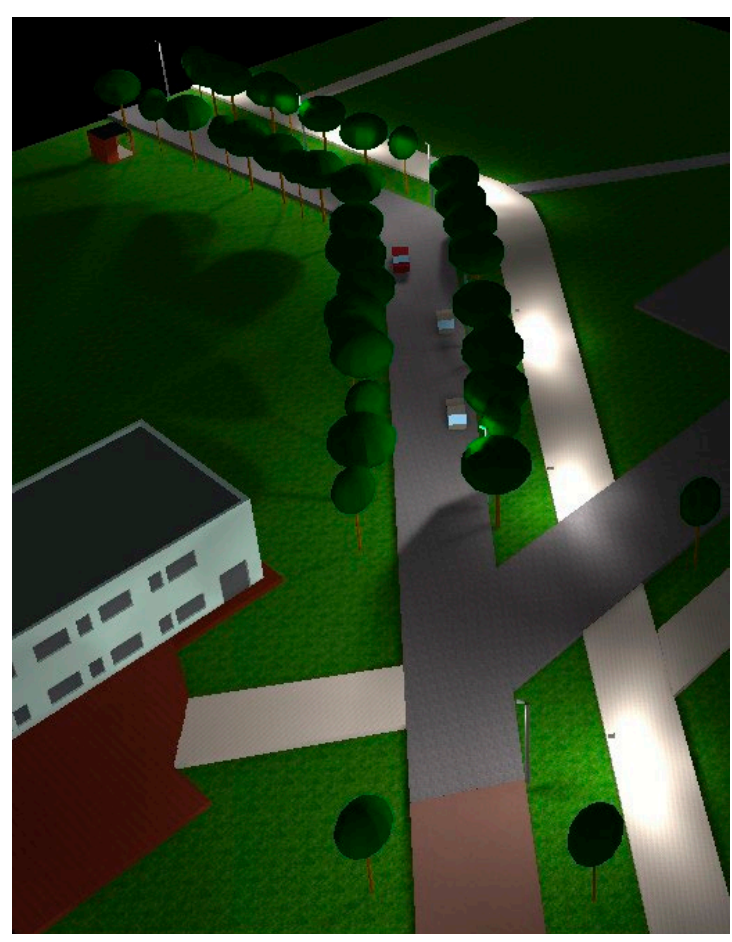

\section{Conclusiones:}

Analizando los datos tabulados en las tablas anteriores y al ser comparados con la tabla suministrada por la norma, se observa que la instalación actual no cumple con los valores mínimos estipulados por la norma (IRAM AADL J2022-2010). Ya que el Emed medido es de1,347, que es inferior al mínimo de vías de clase $\mathrm{F}$, que es de10, según se observa en la tabla que sigue

CARACTERISTICA DEL ALUMBRADO POR EL METODO DE LA ILUMINANCLA

\begin{tabular}{|c|c|c|c|c|}
\hline \multirow{3}{*}{ Clase } & \multicolumn{3}{|c|}{ Valores mínimos } & \multirow{3}{*}{$\begin{array}{l}\text { Grado mínimo de } \\
\text { apantallamiento }\end{array}$} \\
\hline & \multirow{2}{*}{$\begin{array}{l}\text { Nivel inicial } \\
\text { promedio } \\
\text { Emed } \\
(\mathrm{lx})\end{array}$} & \multicolumn{2}{|c|}{ Uniformidad } & \\
\hline & & $\begin{array}{c}\mathrm{G}_{1} \\
\text { Emín / Emed }\end{array}$ & $\begin{array}{c}\mathrm{G}_{2} \\
\text { Emín / Emáx }\end{array}$ & \\
\hline $\mathrm{C}$ & 40 & $1 / 2$ & $1 / 4$ & APANTALLADO \\
\hline $\mathrm{D}$ & 27 & $1 / 3$ & $1 / 6$ & SEMIAPANTALLADO \\
\hline $\mathrm{E}$ & 16 & $1 / 4$ & $1 / 8$ & SEMIAPANTALLADO \\
\hline $\mathbf{F}$ & 10 & $1 / 4$ & $1 / 8$ & NO APANTALLADO \\
\hline
\end{tabular}

Discusión de resultados: Como alternativa se propone una solución coherente y de simple ejecución, la cual propone un cambio de las luminarias actuales por luminarias de mejor rendimiento lumínico, con tecnología led (LG LED street light $180 \mathrm{w} 570 \mathrm{~K}$ type V) que fue seleccionada por su mejor performance. También se plantea reforzar la iluminación sin la necesidad de hacer una nueva potación, colocando sobre la ya existente una luminaria a una menor altura y del lado de la calzada sostenida por un soporte con la 
misma inclinación que el superior, pero una Para la vía de circulación de peatones se longitud de brazo menor; la luminaria suge- propone la misma luminaria (abaligth LED rida es (abaligth LED 60w IP65) extraído 60w IP65) a una altura de 4.30metros. del mismo catálogo de la anterior.

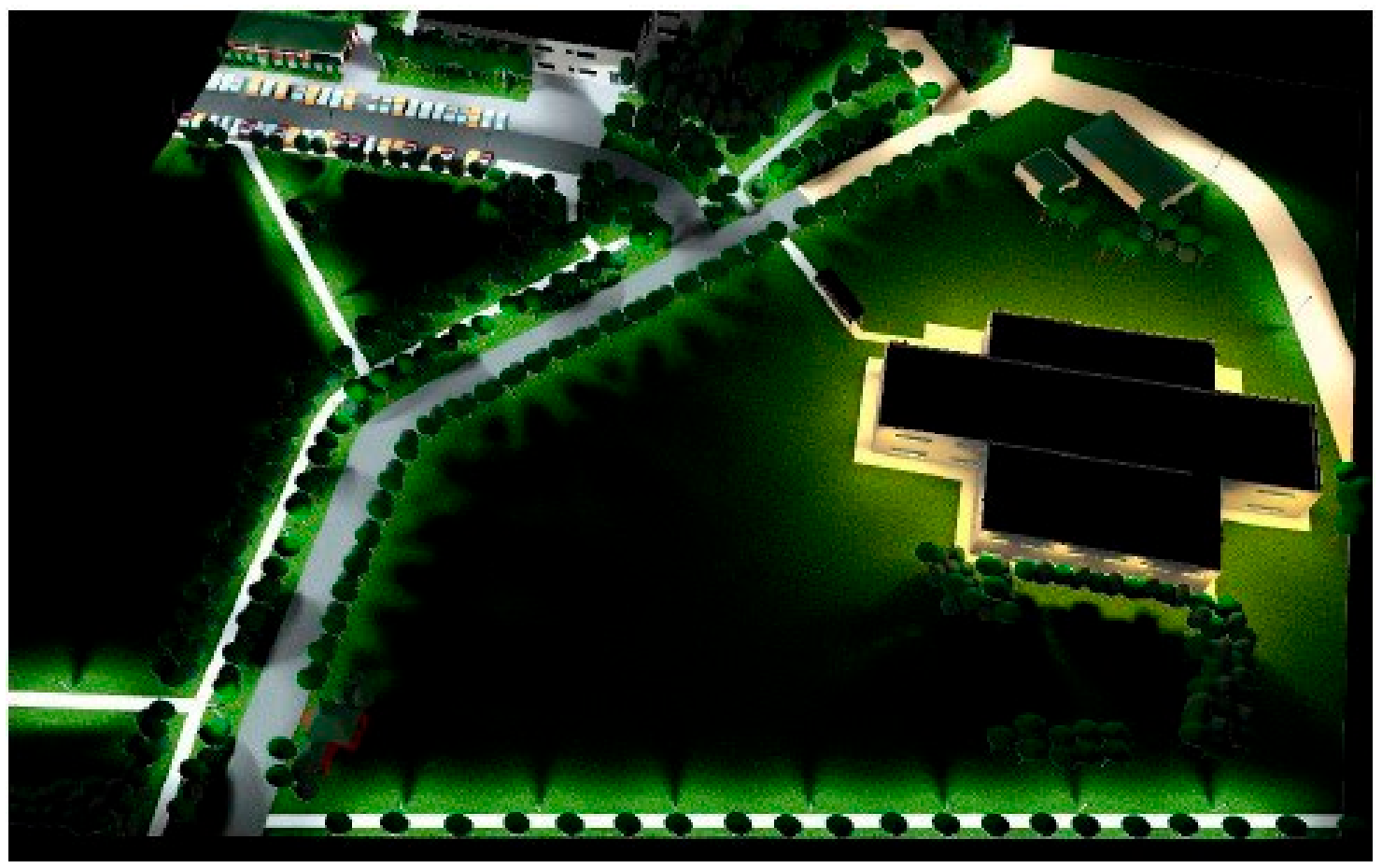

\section{RESULTADOS DE LAS GRILLAS DE CÁLCULO}

TRAMO 1

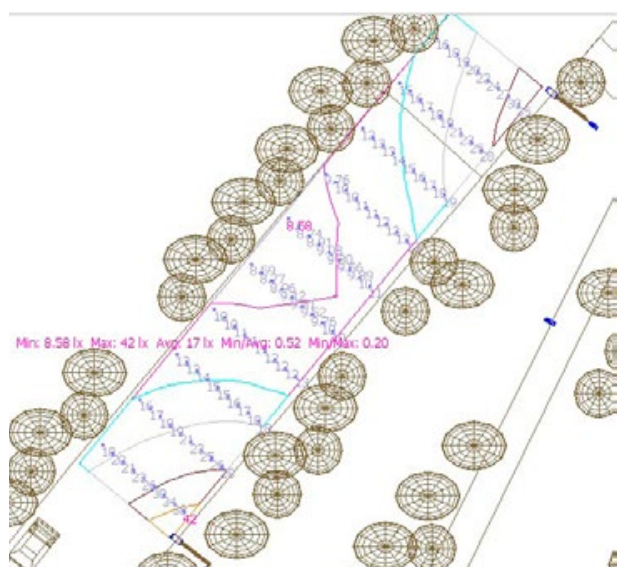

TRAMO 2

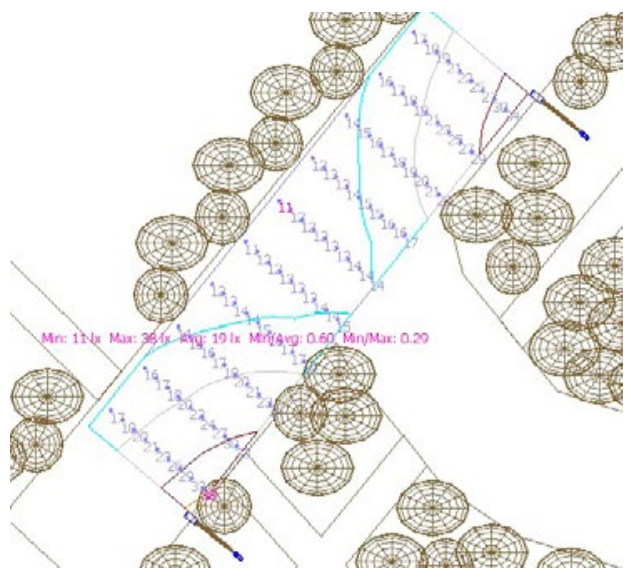




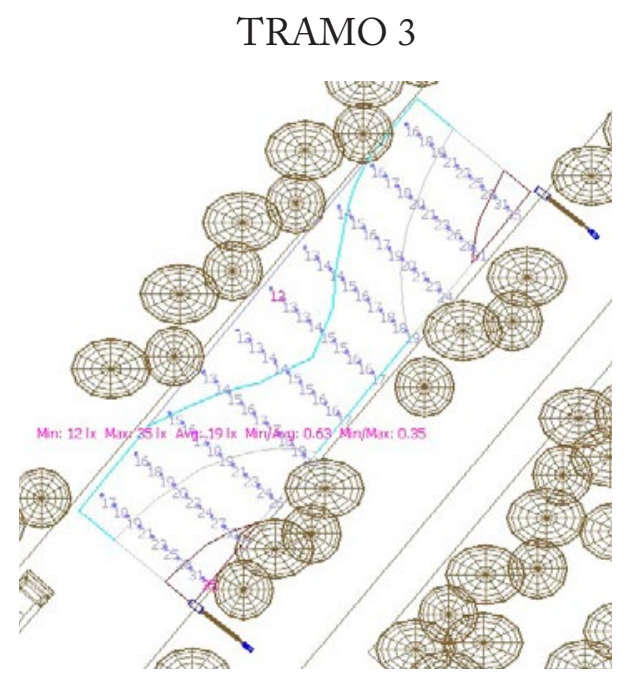

TRAMO 5

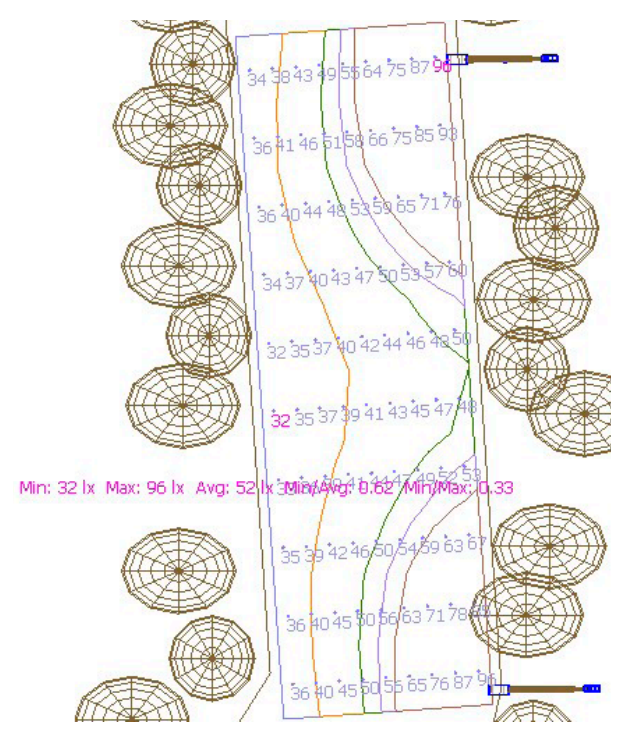

En las imágenes anteriores correspondientes a la alternativa propuestas, se aprecian los valores de Emin, Emax, Em, G1 y G2, los cuales al contrastar se con la tabla de

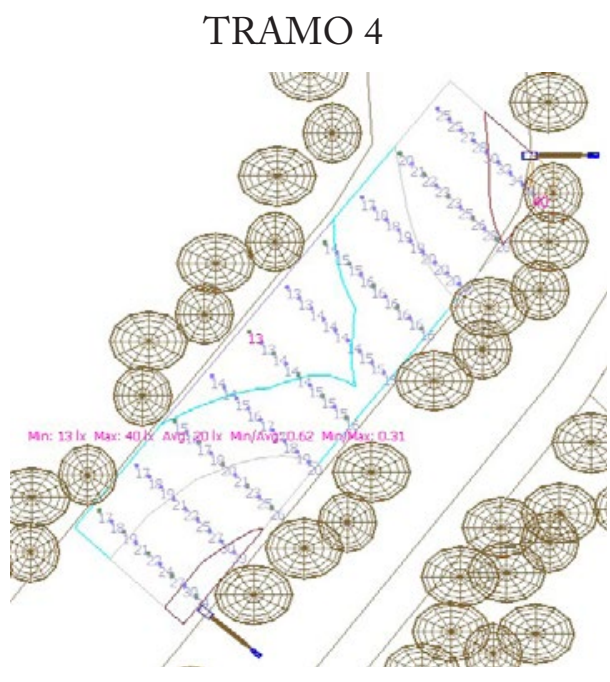

TRAMO 6

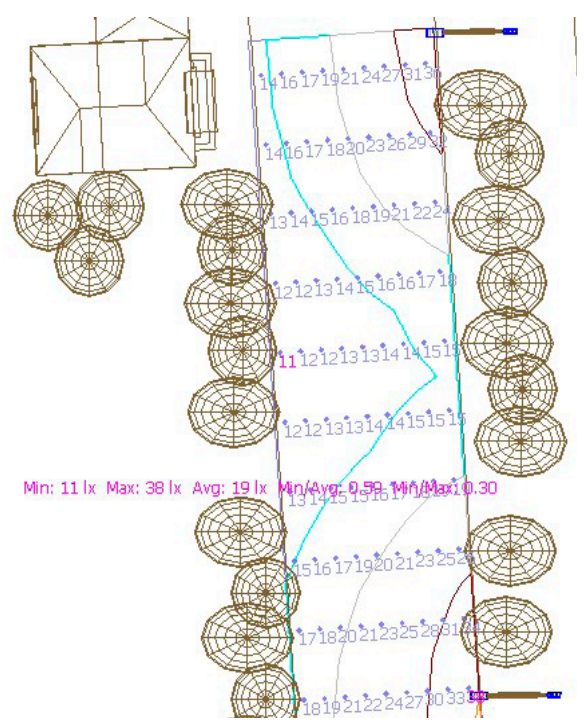

Características de Alumbrado se corrobora que estos valores están dentro de los parámetros determinados por la normativa actualmente vigente en la República Argentina.

\section{BIBLIOGRAFIA:}

- Manual de Luminotecnia - AADL - Tomos I y II.

- NORMA IRAM ADDL J2022-2010 Alumbrado público, vías de tránsito, Parte 2 Clasificación y Niveles de Iluminación.

- Programa de diseño profesional tridimensional en iluminación DIALUX 4.12

- Seip G. Instalaciones Eléctricas -Editorial Siemens.

- Sobrevila M. Instalaciones Eléctricas - Editorial Alsina (Ultima Edición)

- Software de diseño asistido por computadora, 2D y 3D. AUTOCAD 2010, 